\title{
Tangential beam intensity modulated radiotherapy versus tangential beam three-dimensional conformal radiotherapy in carcinoma breast: A dosimetric comparison and clinical correlation
}

\author{
Deepti Arun Phansopkar, Jaineet Sachdeva, Manmohan Krishan Mahajan, Pamela Alice Kingsley, \\ Shekhar Upadhyay, Rajesh Chakravarti \\ Department of Radiation Oncology, Christian Medical College and Hospital (CMC), Ludhiana, India
}

Purpose: This study evaluates the dose distribution of tangential beam intensity modulated radiotherapy (IMRT) compared to tangential beam three-dimensional conformal radiotherapy (3D-CRT) in carcinoma breast.

Methods: Tangential beam IMRT and tangential beam 3D-CRT plans were generated in 54 postoperative breast cancer patients. Planning target volume (PTV) definition of chest wall and intact breast was done according to Breast Cancer Atlas for Radiation Therapy Oncology Group. The prescribed dose was $50 \mathrm{~Gy}$ in 25 fractions. Dose volume histograms was evaluated for PTV and organs at risk, parameters of the dose distribution were compared for 3D-CRT and IMRT using Wilcoxon Matched pairs test. The dosages were clinically correlated with normal tissue reactions.

Results: No difference in the conformity index but IMRT improves the homogeneity index significantly $(P<0.01)$. The mean dose to all organs at risk was lesser in IMRT as compared to 3D-CRT of which the dose to contralateral lung was significantly lower with IMRT (111.7 cGy vs. $41.98 \mathrm{cGy}, \mathrm{P}=0.024)$. Tangential beam IMRT significantly reduces ipsilateral lung V20\% by $24 \%$. Heart V70\% is decreased by V74\% and V30\% by V38\% doses but not statistically significant.

Conclusion: Tangential beam IMRT in breast cancer offers the potential to significantly reduce the dose-volume of the ipsilateral lung and dose volume of the heart which will translate into decrease in risk of cardiac and lung toxicity and also decreased second cancer risk. IMRT definitely decreases the acute skin reactions in all patients.

Keywords: Breast cancer, Intensity modulated radiotherapy, Three-dimensional conformal radiotherapy, Dosimetric comparison

\section{INTRODUCTION}

Breast cancer is a major public health problem worldwide. It is the most frequent cancer with 1.05 million new cases diagnosed every year and represents over 20\% of all malignancies among females [1]. The incidence of breast cancer has steadily increased in India

Received: Aug 4, 2015 Accepted: Oct 26, 2015

Correspondence to: Deepti Arun Phansopkar

Department of Radiation Oncology, Christian Medical College \& Hospital (CMC), Brown Road, Ludhiana 141008, Punjab, India

Tel: +91-964-620-0272, +91-9920821933

E-mail: deeptiphansopkar26feb@gmail.com

Copyright ( $($ Korean Society of Surgical Oncology

This is an Open Access article distributed under the terms of the Creative Commons Attribution Non-Commercial License (http://creativecommons.org/licenses/by-nc/3.0) which permits unrestricted non-commercial use, distribution, and reproduction in any medium, provided the original work is properly cited. over the years and as many as 100,000 new patients are being detected every year [2]. Adjuvant radiation after breast conserving surgery decreases the risk of local recurrence by two-third and results in survival equivalent to that achieved by patients treated with mastectomy [3]. Most mastectomy series show that more than $50 \%$ of loco regional failures (LRF) occur on the chest wall, with the mastectomy scar at greatest risk for recurrence. Therefore, treatment to the chest wall is recommended for almost all post mastectomy patients. The second most common site of LRF is the supraclavicular/infra clavicular (axilllary apex) region. As many as $33 \%$ of LRF occur in this region, with absolute rates of first failure reported in up to $18 \%$ of patients, depending upon extent of axilllary involvement and tumor size $[4,5]$. Post mastectomy radiotherapy includes treatment to the chest wall and draining lymphatics. The most commonly used technique to treat the whole breast is 
currently called a tangent beam. The tangential beam includes part of the anterior thoracic cavity, thereby, potentially affecting the organs at risk (OAR), in particular the lung and heart. Due to the curvature of the breast and the chest wall, some volume of lung (and the heart in left-sided breast cancers) is included in the radiation beam. Randomized, retrospective and population based studies have shown that radiotherapy of chest wall is associated with significantly increased risk of developing ipsilateral second lung cancer [6] and in patients treated on left side with significantly increased risk of cardiac morbidity and mortality [7].

Clinically it is not possible to estimate the effect of heart dose volume reduction by the tangential beam intensity modulated radiotherapy (IMRT). Radiation associated heart disease involves a spectrum of clinical diagnosis including pancarditis, pericarditis, cardiomyopathy and coronary heart disease with ischeamic heart disease. Clinical presentations of radiation induced heart disease have been observed in patients who received therapeutic doses of about $\geq 35$ Gy to partial volumes of the heart [8]. Predisposing factors for radiation induced heart disease are pre-existing cardiovascular disease, smoking, obesity, and hypertension as well as the use of cardiotoxic agents such as anthracycline, paclitaxel and trastuzumab. The long-term risk of dying of cardiac disease is of particular concern for women treated for left sided breast cancer with contemporary tangential breast or chest wall radiotherapy. The risk may increase with a longer follow up, even after 20 years following radiotherapy [9]. An increased risk of secondary tumours has been observed in breast cancer patients treated with older radiation techniques, which combined higher radiation dose and larger tissue volumes. The carcinogenic effects of radiotherapy used for the treatment of breast cancer patients have recently been reviewed. Second malignancies develop in the radiation field in a small number of cases. The two types of potentially lethal malignancy that appear in the radiation field most often are soft tissue and bone sarcomas and lung cancer [10].

A variety of dosimetric studies have suggested that IMRT potentially leads to a more favorable dose distribution compared to three-dimensional planned conformal radiotherapy (3D-CRT) for the radiotherapy of the whole breast after breast conserving surgery [11]. Radiotherapy treatment planning for the chest wall is complex due to missing tissue and the presence of lung tissue within the treatment field. Accurate dose distribution is important as doses to neighboring OAR, such as heart, lungs and contralateral breast, needs to be minimized [12].

There are many differences between the target volume of the chest wall and the whole breast. The shape of the target volume of the chest wall is usually shallower compared to the whole breast. In addition, in stage I-IIA patients the pectoralis muscle, chest wall muscles and ribs may be excluded in the target volume of the whole breast, whereas these structures are included in the target volume of the chest wall. Due to these differences in the target volume, results of a dosimetric study of the radiotherapy of the whole breast may not be completely applicable to the radiotherapy of the chest wall. Thus, a dosimetric study of the chest wall and whole breast is required to compare IMRT with 3D-CRT in postoperative breast cancer patients.

This study has been designed to evaluate the dose distribution of tangential beam forward planning IMRT in breast cancer patients compared to tangential beam 3D-CRT and correlate the same with normal tissue toxicity.

\section{METHODS}

Fifty four postoperative breast cancer patients coming to a tertiary care center were included in the study out of which 44 patients underwent modified radical mastectomy and breast conservative surgery conservation surgery in 10 patient. A tangential beam IMRT plan and a standard tangential beam 3D-CRT plan were generated. Twenty one patients had right-sided breast cancer and thirty-two had left-sided, while one patient had bilateral breast cancer. The target volumes and the dose prescribed according to the International Commission on Radiation Units and Measurement Reports 50 and 62 recommendations. According to this target volume should be surrounded by the $95 \%$ isodose line. The planning target volume (PTV) for chest wall and intact breast was defined according to the Breast Cancer Atlas for radiation therapy planning consensus definitions of the Radiation Therapy Oncology Group. The chest wall with the pectoralis muscle, chest wall muscles, and ribs, were included in PTV and excluded the outermost $3 \mathrm{~mm}$ from the superficial skin surface. PTV of the intact breast included apparent computed tomography (CT) glandular breast tissue and excluded the chest wall, pectoralis muscle, ribs, skin with additional 3D margin of $5 \mathrm{~mm}$ around the breast clinical target volume (CTV) The heart was defined as all visible myocardium, from the apex to the right auricle, atrium, and infundibulum of the ventricle. The pulmonary trunk, root of the ascending aorta, and superior vena cava were excluded.

\section{Treatment techniques}

A non-contrast CT-simulation was performed in the supine position on a breast board with the ipsilateral arm up and head turned to the contralateral side. Radio-opaque wires were used to mark the mastectomy scar and the clinical boundaries (inferior aspect of clavicular head, mid sternum, mid axillary line, $2 \mathrm{~cm}$ below the level of the contralateral infra-mammary sulcus). A spiral CT scan was performed using $3 \mathrm{~mm}$ slice thickness. The CT scanning reference point 
and PTV and the OAR were defined. Using Focal Workstation. The 3D-CRT and IMRT plans were generated using Treatment Planning System CMS XiO 4.6.

An Elekta linear accelerator with single photon energy of $6 \mathrm{MV}$ and multileaf collimator was used for the treatment. The leaf width was $1 \mathrm{~cm}$ from the isocenter. The dose calculation was determined using the "Superposition" algorithm. The prescribed total dose was 50 Gy in 25 fractions for 5 weeks for all patients. The beam energy of $6 \mathrm{MV}$ was used for all 3D-CRT and IMRT plans. Both the 3D-CRT and forward planning IMRT plans were generated on the PTV of each patient and dose parameters were compared. The plan with the better Homogeneity Index, Conformity Index and decreased normal tissue dosages was taken as the better plan and was executed on the patient.

\section{Statistics}

IMRT and 3D-CRT plan parameters derived from the same patient were tested for statistically significant difference using the Student t-test. Statistical analysis was performed using the Wilcoxon signed rank test. This matched paired t-test was applied to determine the statistical difference between the dose-volume data. The reported 'P' value is two tailed and values of $<0.05$ were considered significant.

\section{Tangential beam 3D-CRT technique}

Two tangential semi-opposed beams (to avoid divergence) and a Multileaf Collimator were used for 3D-CRT. The beam angles, wedge angles, and beam weighting (usually minimal) were chosen to optimize coverage of the PTV, while minimizing exposure to the ipsilateral and contralateral lung, heart and contralateral breast and spinal cord (Fig. 1).

\section{IMRT technique}

The same beam orientation and angles of the 3D-CRT plan were used for the tangential beam of the corresponding IMRT plan. The PTV included the same PTV used for 3D-CRT plan plus an extension into the air anteriorly of the chest of $1.5 \mathrm{~cm}$, to ensure appropriate opening of multileaf collimator. A step-and-shoot technique was applied. The dose was prescribed to the PTV, and as initial dose volume constraints the IMRT prescription table provided by the XiO treatment planning system was used (Table 1). Tissue inhomogeneities were considered in the treatment planning optimization process, and the dose calculation algorithm used was "Superposition".
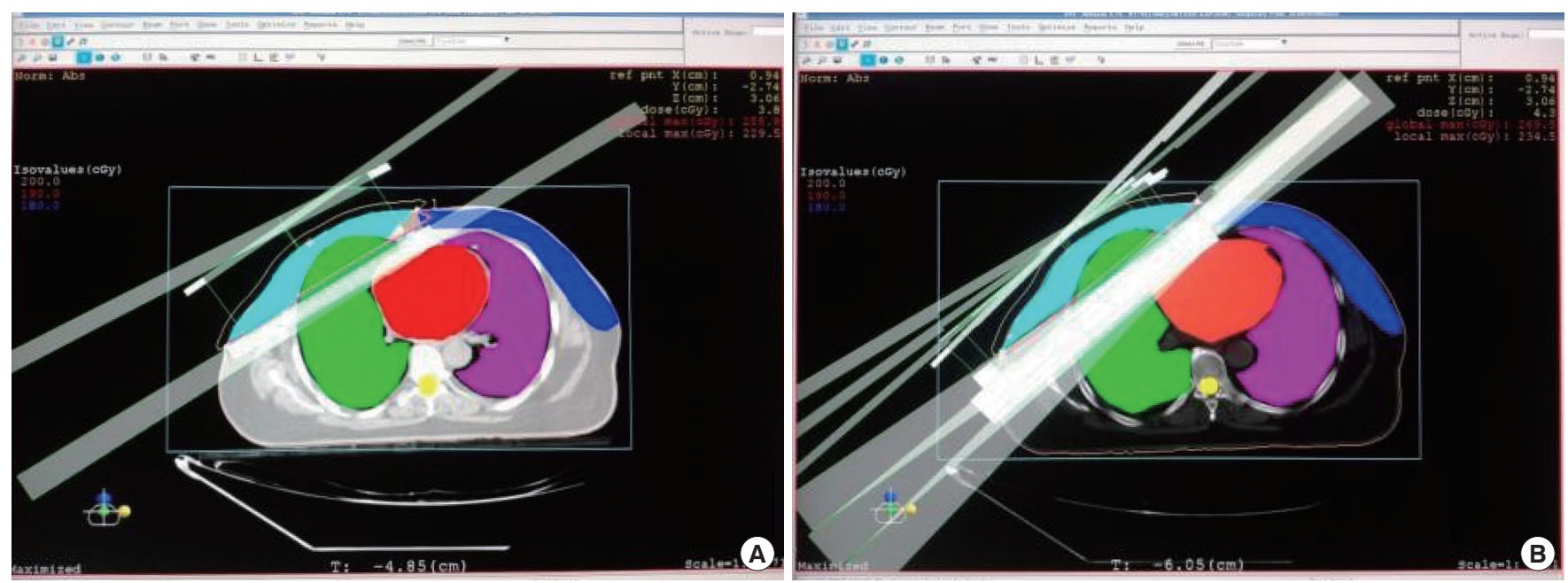

Fig. 1. Demonstrates typical dose distributions of an 3D-CRT and IMRT plan of the same patient. (A) Conformal three-dimensional (3D-CRT) and (B) IMRT plans. 3D-CRT, three-dimensional conformal radiotherapy; IMRT, intensity modulated radiotherapy.

Table 1. Dose volume constraints for intensity modulated radiotherapy plans

\begin{tabular}{llclcrr}
\hline Structure & Type & Rank & Objective & Dose (cGy) & Volume (\%) & Weight \\
\hline Planning target volume & Target & 1 & Maximum & 5,200 & 0 & 100 \\
& Target & 1 & Maximum & 4,900 & 100 & 100 \\
Ipsilateral lung & Organ at risk & 2 & Maximum & 2,000 & 100 & 100 \\
& Organ at risk & 2 & Minimum & 1,200 & 30 & 100 \\
Heart & Organ at risk & 3 & Maximum & 4,500 & 0 & 100 \\
Unspecified tissue & Organ at risk & 4 & Maximum & 4,500 & 100 \\
\hline
\end{tabular}


Dose Volume Histograms were evaluated for the PTV and OAR. The ipsilateral and contralateral lung dose volume, Heart dose volume, contralateral breast dose, spinal cord dose, homogeneity index

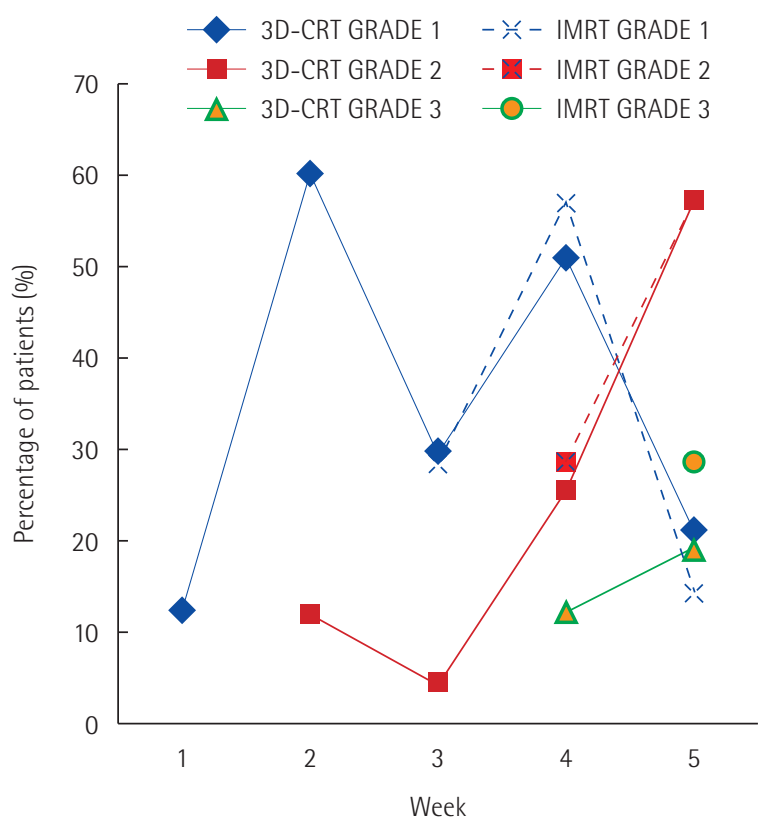

Fig. 2. Distribution according to acute skin reactions on chest wall with 3D-CRT 47 (87.0\%) patients and IMRT 7 (12\%) patients. 3D-CRT, three-dimensional conformal radiotherapy; IMRT, intensity modulated radiotherapy.
(HI), and conformity index $(\mathrm{Cl})$ between IMRT and 3D-CRT groups were compared. The dosimetric volumetric comparison of ipsilateral lung V20\% and heart V70\% and V30\% were studied (Fig. 2).

$\mathrm{HI}$ : defined as the fraction of the PTV with a dose between 95\% and $105 \%$ of the prescribed dose (V95\%-V105\%).

$\mathrm{Cl}$ : defined as the fraction of the PTV surrounded by the reference dose (V95\%) multiplied by the fraction of the total body volume covered by the reference PTV dose (PTV95\%/PTV) × (PTV95\%/ V95\%).

\section{RESULTS}

Tables 2 and 3 compares plan parameters of opposed tangential beam 3D-CRT with Tangential beam IMRT for the adjuvant radiotherapy in 54 postoperative breast cancer patients.

The mean dose to contralateral lung was seen to be significantly higher with 3D-CRT 111.79 cGy vs. 41.98 cGy (IMRT) $P=0.024$. The ipsilateral lung V20\% received significantly higher dose with 3D-CRT than with IMRT (151.2 cGy vs. 115.4 cGy) $P=0.019$.

The $\mathrm{Cl}$ with 3D-CRT was 0.953 vs. 0.951 with IMRT $(P=0.327)$. Tangential beam IMRT improved the Homogeneity index 1.14 vs. $1.193(\mathrm{P}<0.001)$ (Table 4).

In our study, with 3D-CRT in the first week grade I reactions was seen in 1 (12.1\%), second week 3 (60.38\%), third week 14 (29.78\%), fourth week 24 (51.06\%), and fifth week 11 (21.28\%). Grade II reac-

Table 2. Dosimetric comparisons with 3D-CRT and IMRT in breast cancer

\begin{tabular}{|c|c|c|c|c|c|c|}
\hline Dosimetric parameter & Volumes (mL) & 3D-CRT (mean dose, cGy) & Standard deviation & IMRT (mean dose, cGy) & Standard deviation & P-value \\
\hline Ipsilateral lung & $1,090.8$ & $2,669.44$ & $5,661.64$ & $1,437.5$ & 813.2 & 0.109 \\
\hline Contralateral lung & $1,068.58$ & 111.79 & 219.46 & 41.9811 & 41.981 & 0.024 \\
\hline Heart & 481.44 & 781.48 & $1,722.63$ & 431.944 & 357.71 & 0.140 \\
\hline Contralateral breast & 868.64 & 323.611 & 899.664 & 98.1481 & 119.437 & 0.071 \\
\hline Spinal cord & 28.675 & 74.537 & 62.19 & 33.333 & 12.848 & 0.068 \\
\hline
\end{tabular}

3D-CRT, three-dimensional conformal radiotherapy; IMRT, intensity modulated radiotherapy.

Table 3. Dose volumetric parameters with 3D-CRT and IMRT in breast cancer

\begin{tabular}{lccccc}
\hline Dosimetric parameter & 3D-CRT (mean dose, cGy) & Standard deviation & IMRT (mean dose, cGy) & Standard deviation & P-value \\
\hline Ipsilateral lung V20\% & 151.26 & 359.72 & 115.44 & 52.60 & 0.019 \\
Heart V70\% & 10.52 & 37.67 & 2.76 & 3.824 & 0.108 \\
V30\% & 30.56 & 55.88 & 19.12 & 10.90 & 0.806 \\
\hline
\end{tabular}

3D-CRT, three-dimensional conformal radiotherapy; IMRT, intensity modulated radiotherapy.

Table 4. Dosimetric comparison of conformity and homogeneity index in 3D-CRT and IMRT in breast cancer

\begin{tabular}{lccccr}
\hline Dosimetric parameter & 3D-CRT & Standard deviation & IMRT & Standard deviation & P-value \\
\hline Conformity index & 0.953 & 0.00964 & 0.951 & 0.0089 & 0.327 \\
Homogeneity index & 1.143 & 0.0570 & 1.193 & 0.0696 & $<0.001$ \\
\hline
\end{tabular}

3D-CRT, three-dimensional conformal radiotherapy; IMRT, intensity modulated radiotherapy. 
tions in second week $1(12.12 \%)$, third week $2(4.26 \%)$, fourth week $12(25.53 \%)$, fifth week $27(57.4 \%)$, while grade III was seen in the fourth week in 1 (12.12\%), and fifth week 9 (19.14\%).

While with IMRT grade I reactions appeared only in the third week in 2 (28.5\%) patients, fourth week 4 (57.12\%), fifth week 1 (14.2\%). But by end of fifth week, smooth grade II reactions were seen in 4 patients and grade III in $2(28.57 \%)$ patients, respectively.

Onset of reactions were faster in 3D-CRT versus IMRT (first week versus third week). However at the end of treatment almost equal number of patients had grade II-III reactions. Reactions were smooth over the chest wall in both technique.

\section{DISCUSSION}

Several studies have showed a dosimetric benefit of IMRT compared to 3D-CRT for the whole breast in early breast cancer patients. Data about the impact of IMRT on the adjuvant radiotherapy of the chest wall in post mastectomy patients are scarce in the literature. In our country patients come in advanced stages when breast conserving surgery is not possible. So mastectomy is the treatment of choice here. There are distinct geometric differences between the target volume of the chest wall and the whole breast, and these differences might have an impact on the resulting dose distribution. This study was undertaken to evaluate the dose distribution of tangential beam forward planning IMRT versus Tangential Beam 3D-CRT. In our study, there was no difference in the $\mathrm{Cl}$ but IMRT improves the $\mathrm{HI}$ significantly $(\mathrm{P}<0.01)$.

The mean dose to all OAR was lesser in IMRT as compared to 3D-CRT of which the dose to contralateral lung was significantly lower with IMRT (111.7 cGy vs. $41.98 \mathrm{cGy}, \mathrm{P}=0.024$ ).

However, the doses to ipsilateral lung, heart, contralateral breast and spine, though lower with IMRT, were not statistically significant. Our data show that tangential beam IMRT compared to 3D-CRT significantly reduces Ipsilateral Lung V20\% by $24 \%$, Heart V70\% by $74 \%$ and $\mathrm{V} 30 \%$ by 38\% doses than 3D-CRT, but were not statistically significant.

Similar results have been reported by Selvaraj et al. [13] compared IMRT based treatment plan with 3D-CRT. DHI was increased by 6.3\% with IMRT compared to 3D-CRT $(P<0.05)$. The mean dose and $\mathrm{V}_{30}$ for heart with IMRT were 2.3 (standard deviation [SD], 1.1) and $1.05(\mathrm{SD}, 1.5)$ respectively, which was a reduction by $6.8 \%$ and $7.9 \%$, respectively, in comparison with 3D-CRT. Similarly, the mean dose and $V_{20}$ for the ipsilateral lung and the percentage of volume of contralateral volume lung receiving $>5 \%$ of prescribed dose with IMRT were reduced by $9.9 \%, 2.2 \%$, and $35 \%$, respectively. Their dosimetric data suggested improved dose homogeneity in the breast and reduction in the dose to lung and heart with IMRT.
Rudat et al. [14] reported IMRT resulted in significantly decreased heart and lung high dose volume with a significantly improved $\mathrm{Cl}$ when compared to 3D-CRT. They also found that tangential beam IMRT for irradiation of the chest wall of post mastectomy breast cancer patients could significantly decrease the dose-volume of the ipsilateral lung.

Smith et al. [15] compared three tangential beam IMRT plans with conventional tangential beam $2 \mathrm{D}$ plans for the adjuvant radiotherapy of the whole breast in 20 patients with early breast cancer. All IMRT plans showed a significant improvement of the PTV $\mathrm{HI}$ of $15 \%$, heart $\mathrm{V} 30 \%$ of $28 \%$ to $33 \%$, and whole lung $\mathrm{V} 20 \%$ of $2 \%$ to $8 \%$ compared to the conventional technique.

Moorthy et al. [16] compared and analyzed the dosimetric aspects of IMRT over 3D-CRT for chest wall. There was a consistent improvement in $\mathrm{Cl}$ for breast volume from 1.33 for 3D-CRT to 1.15 for IMRT $(P<0.05)$. The Homogeneity Index was improved with IMRT for the breast volume $(P<0.05)$. Ipsilateral lung V20 Gy was significantly reduced with IMRT $(P<0.01)$. Both lungs V20 Gy was significantly improved with IMRT $(P<0.01)$. The heart V30 Gy was $1.12 \%$ in IMRT against 2.5\% in 3D-CRT. They concluded that both the methods achieved adequate target coverage, IMRT reduces maximum doses and improves Conformity and Homogeneity indices of target volumes, also reduces dose to OAR.

Freedman et al. [17] reported that IMRT decreases Acute Skin Toxicity for Women Receiving Radiation for Breast Cancer. There was no desquamation in $42 \%$ of IMRT patients, dry desquamation in 37\% and moist desquamation in 21\%. The degree of desquamation was greater for conventional patients compared with IMRT patients $52 \%$ grade $0,10 \%$ grade 1 , and $38 \%$ grade $2(P=0.001)$.

Prospective studies with long follow-up times are needed to fully evaluate the cardiac toxicity and secondary lung cancer risk in breast cancer patients treated with tangential beam IMRT.

Tangential beam IMRT for the radiotherapy in breast cancer patients offers the potential to significantly reduce the dose-volume of the ipsilateral lung, dose-volume of the heart compared to tangential beam 3D-CRT which will translate into decrease in risk of cardiac and lung toxicity also decreased second cancer risk. IMRT definitely delays the acute skin reactions in all patients.

\section{CONFLICT OF INTEREST}

No potential conflict of interest relevant to this article was reported.

\section{REFERENCES}

1. Parkin DM, Bray F, Ferlay J, Pisani P. Estimating the world cancer burden: Globocan 2000. Int J Cancer 2001;94:153-6. 
2. Zeleniuch JA, Roy ES. Epidemiology of breast cancer. In: Roses DF, editor. Breast cancer. 2nd ed. Philadelphia: Elsevier Churchill Livingstone; 2005. p.3-14.

3. Fisher $B$, Anderson $S$, Redmond CK, Wolmark N, Wickerham DL, Cronin WM. Reanalysis and results after 12 years of follow-up in a randomized clinical trial comparing total mastectomy with lumpectomy with or without irradiation in the treatment of breast cancer. N Engl J Med 1995;333:1456-61.

4. Nguyen-Tan PF, Vincent L, Methot $F$, Bahary JP. The incidence of supraclavicular failure in patients with T1-2 breast cancer and four or more positives nodes treated by conservative surgery and tangential breast irradiation without regional nodal irradiation. Int J Radiat Oncol Biol Phys 1998;42:249.

5. Schwaibold F, Fowble BL, Solin U, Schultz DJ, Goodman RL. The results of radiation therapy for isolated local regional recurrence after mastectomy. Int J Radiat Oncol Biol Phys 1991;21:299-310.

6. Berrington de Gonzalez A, Curtis RE, Gilbert E, Berg CD, Smith SA, Stovall $M$, et al. Second solid cancers after radiotherapy for breast cancer in SEER cancer registries. Br J Cancer 2010;102:220-6.

7. Giordano SH, Kuo YF, Freeman JL, Buchholz TA, Hortobagyi GN, Goodwin JS. Risk of cardiac death after adjuvant radiotherapy for breast cancer. J Natl Cancer Inst 2005;97:419-24.

8. Doyen J, Giraud P, Belkacemi Y. Normal tissue tolerance to external beam radiation therapy: cardiac structures. Cancer Radiother 2010;14:319-26.

9. Bouillon K, Haddy N, Delaloge S, Garbay JR, Garsi JP, Brindel P, et al. Long-term cardiovascular mortality after radiotherapy for breast cancer. J Am Coll Cardiol 2011;57:445-52.

10. Roychoudhuri R, Evans H, Robinson D, Moller H. Radiation-in- duced malignancies following radiotherapy for breast cancer. $\mathrm{Br} J$ Cancer 2004;91:868-72.

11. Barnett GC, Wilkinson J, Moody AM, Wilson CB, Sharma R, Klager $\mathrm{S}$, et al. A randomised controlled trial of forward-planned radiotherapy (IMRT) for early breast cancer: baseline characteristics and dosimetry results. Radiother Oncol 2009;92:34-41.

12. Ahnesjo A, Knoos T, Montelius A. Application of the convolution method for calculation of output factors for therapy photon beams. Med Phys 1992;19:295-301.

13. Selvaraj RN, Beriwal S, Pourarian RJ, Lalonde RJ, Chen A, Mehta K, et al. Clinical implementation of tangential field intensity modulated radiation therapy (IMRT) using sliding window technique and dosimetric comparison with 3D conformal therapy (3DCRT) in breast cancer. Med Dosim 2007;32:299-304.

14. Rudat V, Alaradi AA, Mohamed A, Ai-Yahya K, Altuwaijri S. Tangential beam IMRT versus tangential beam 3D-CRT of the chest wall in postmastectomy breast cancer patients: a dosimetric comparison. Radiat Oncol 2011;6:26.

15. Smith W, Menon G, Wolfe N, Ploquin N, Trotter T, Pudney D. IMRT for the breast: a comparison of tangential planning techniques. Phys Med Biol 2010;55:1231-41.

16. Moorthy S, DasMajumdar SK, Elhateer H, Mohan R, Mohammed S, Shaima, et al. Dosimetric analysis of IMRT versus 3DCRT for chest wall irradiation in patients with breast cancer using $6 \mathrm{MV} \mathrm{X}$-rays. Indian J Res Rep Med Sci 2013;3:36-9.

17. Freedman GM, Anderson PR, Li J, Eisenberg DF, Hanlon AL, Wang L, et al. Intensity modulated radiation therapy (IMRT) decreases acute skin toxicity for women receiving radiation for breast cancer. Am J Clin Oncol 2006;29:66-70. 